\title{
Relation between the Migdal Effect and Dark Matter-Electron Scattering in Isolated Atoms and Semiconductors
}

\author{
Rouven Essig, ${ }^{1, *}$ Josef Pradler, ${ }^{2, \dagger}$ Mukul Sholapurkar, ${ }^{1, \$}$ and Tien-Tien Yu ${ }^{3, \S}$ \\ ${ }^{1}$ C.N. Yang Institute for Theoretical Physics, Stony Brook University, Stony Brook, New York 11794, USA \\ ${ }^{2}$ Institute of High Energy Physics, Austrian Academy of Sciences, Nikolsdorfergasse 18, 1050 Vienna, Austria \\ ${ }^{3}$ Department of Physics, University of Oregon, Eugene, Oregon 97403, USA
}

(Received 30 August 2019; published 13 January 2020)

\begin{abstract}
A key strategy for sub-GeV dark matter direct detection is searches for small ionization signals that arise from dark matter-electron scattering or from the "Migdal" effect in dark matter-nucleus scattering. We show that the theoretical description of both processes is closely related, allowing for a principal mapping between them. We explore this for noble-liquid targets and, for the first time, estimate the Migdal effect in semiconductors using a crystal form factor. We present new constraints using XENON10, XENON100, and SENSEI data, and give projections for proposed experiments.
\end{abstract}

DOI: 10.1103/PhysRevLett.124.021801

Introduction.-Detectors searching for direct signals from dark matter (DM) are conventionally optimized to look for electroweak-scale DM that scatters elastically off atomic nuclei. The detectors typically lose sensitivity rapidly for $\mathrm{DM}$ masses below a few $\mathrm{GeV}$, due to an inefficient energy transfer from the DM to the recoiling nucleus. However, the kinematic limitations are lifted when the DM-nucleus scattering process is accompanied by the irreducible simultaneous emission of a "bremsstrahlung" photon [1] or a "Migdal" electron [2], or by considering alternative interactions such as DM-electron scattering [3]. In all cases the entire energy of relative motion between the atom and DM can in principle be transferred to the outgoing photon or electron. These signals have already opened a new pathway for current detectors to register DM scattering on nuclei [4-7] for DM masses below $100 \mathrm{MeV}$ (driven primarily by the stronger Migdal effect), and DM scattering on electrons [8-16] for masses as low as $500 \mathrm{keV}$. The sensitivity to sub-GeV DM is expected to improve significantly over the next few years as new detectors with an ultralow ionization threshold are being developed $[10,17]$. For related and distinct direct-detection ideas to probe sub-GeV DM; see, e.g., Refs. [18-48].

The theoretical description of the bremsstrahlung and Migdal effect is so far exclusively tied to a picture where DM scatters on a single, isolated atom [1,2], which for inner-shell electrons should provide a correct estimate of the expected signal rate. For the outer-shell electrons, one might have

Published by the American Physical Society under the terms of the Creative Commons Attribution 4.0 International license. Further distribution of this work must maintain attribution to the author(s) and the published article's title, journal citation, and DOI. Funded by SCOAP ${ }^{3}$. some hope that the isolated atom calculations are still valid for noble liquids, but the complicated electronic band structure for semiconductor targets definitely calls for a different approach. The results presented for noble liquids in Refs. [4,7] and for germanium in Refs. [5,6] restricted themselves to inner-shell electrons and had higher detector threshold than those needed to see a dominant signal from the outer shells. However, several experiments already have sensitivity to one or a few electrons, and a complete understanding of the Migdal and bremsstrahlung effect will maximize our sensitivity to nuclear scattering. On the other hand, DM-electron scattering has already been calculated with a detailed accounting of the band structure in Ref. [22].

In this work, our first goal is to show that the theoretical calculation of the rates for the Migdal effect and for DM-electron scattering are closely related. This observation allows us to take the first steps towards calculating the Migdal effect in semiconductors, thereby overcoming the previous limitations in the theoretical description. It allows us also to calculate the first DM-nucleus scattering limits down to DM masses of $500 \mathrm{keV}$ using published SENSEI results with a single-electron threshold [12]. A second goal is to use the observed single- and few-electron events from XENON10 to calculate the Migdal effect, which provides a DM-nuclear scattering limit down to $5 \mathrm{MeV}$, lower in mass than other published constraints. We compare this limit to XENON100 and XENON1T. Finally, a third goal is to contrast the constraints from DM-electron scattering with those from the Migdal effect in DM-nuclear scattering when the DM interations with ordinary matter are mediated by a dark photon, which gives rise to both signals simultaneously. A more detailed exposition will be provided in a companion paper [49].

To appreciate the connection between the DM-electron and Migdal processes, consider the nucleus receiving a sudden 
three momentum transfer $\boldsymbol{q}$ in the scattering with DM. The probability for promptly ejecting an electron from an atom that was initially at rest in state $|i\rangle$ is then found by projecting the boosted electron cloud onto the desired final state $|f\rangle$,

$$
\left\langle f\left|e^{i\left(m_{e} / m_{N}\right) \boldsymbol{q} \cdot \sum_{\alpha} \boldsymbol{x}^{(\alpha)}}\right| i\right\rangle \simeq \frac{i}{e} \frac{m_{e}}{m_{N}} \boldsymbol{q} \cdot \boldsymbol{d}_{f i} \quad(i \neq f) .
$$

Here, $m_{e}\left(m_{N}\right)$ is the mass of the electron (nucleus) and the sum runs over all electron positions $\boldsymbol{x}^{(\alpha)}$. The exponential on the left-hand side is indeed the electron boost operator as $\boldsymbol{v}_{N}^{\prime}=\boldsymbol{q} / m_{N}$ is the velocity of the recoiling nucleus. On the right-hand side, we have used the dipole approximation, noting that $\left(m_{e} / m_{N}\right) \boldsymbol{q} \cdot \boldsymbol{x}^{(\alpha)} \ll 1$ in all cases of interest; $\boldsymbol{d}_{f i}=\left\langle f\left|e \sum_{\alpha} \boldsymbol{x}^{(\alpha)}\right| i\right\rangle$ is the atom transition dipole moment. In single-electron transitions only one term will contribute in the latter sum, and $\boldsymbol{d}_{f i}$ is to be replaced by $\boldsymbol{d}_{f i} \rightarrow \boldsymbol{d}_{f i}^{(\beta)}=$ $\left\langle f\left|e \boldsymbol{x}^{(\beta)}\right| i\right\rangle$, where $\boldsymbol{x}^{(\beta)}$ is the coordinate of the electron undergoing the transition.

In turn, in the calculation of the ionization probability from the direct interaction of DM with an electron at coordinate $\boldsymbol{x}^{(\beta)}$, the following matrix element enters,

$$
\left\langle f\left|e^{i \boldsymbol{q} \cdot \boldsymbol{x}^{(\beta)}}\right| i\right\rangle \simeq \frac{i}{e} \boldsymbol{q} \cdot \boldsymbol{d}_{f i}^{(\beta)} \quad(i \neq f) .
$$

Here the momentum transfer $\boldsymbol{q}$, i.e., the momentum lost by the DM particle, is directly picked up by the electron. Comparing Eq. (1) with Eq. (2) clearly exposes the similarity of Migdal-effect and DM-electron scattering. However, at the same time this also shows that the atom is probed at vastly different momentum transfers in the respective processes, a factor of $\sim 10^{-3} / A$ softer in the Migdal case.

The Migdal effect in isolated atoms.-We now establish the precise relation between DM-electron scattering and Migdal effect for isolated atoms. In an isolated atom, the bound initial-state electron wave functions are specified by their principal and orbital quantum numbers $n$ and $l$. We are interested in transitions to continuum final states that are characterized by momentum $p_{e}=\sqrt{2 m_{e} E_{e}}$ and orbital quantum number $l^{\prime}$. We define the ionization probability for such a scenario as

$$
\left|\left\langle p_{e}, l^{\prime}\left|e^{i\left(m_{e} / m_{N}\right) \boldsymbol{q} \cdot \sum_{\alpha^{x}} \boldsymbol{x}^{(\alpha)}}\right| n, l\right\rangle\right|^{2}=\frac{1}{2 \pi} \frac{d p_{n l \rightarrow E_{e}}(q)}{d E_{e}} .
$$

A key observation of this Letter is that the electronionization probability $d p_{n l \rightarrow E_{e}}(q) / d E_{e}$ is related to a dimensionless form factor $\left|f_{n l}^{\text {ion }}\left(p_{e}, q_{e}\right)\right|^{2}$ that was previously defined in the context of DM-electron scattering $[3,8]$. Concretely, we find

$$
\frac{d p_{n l \rightarrow E_{e}}}{d \ln E_{e}}=\frac{\pi}{2}\left|f_{n l}^{\text {ion }}\left(p_{e}, q_{e}\right)\right|^{2},
$$

where the form factor is evaluated at $q_{e} \simeq\left(m_{e} / m_{N}\right) q$. Capturing the Migdal process hence becomes a question of calculating the form factor accurately. It is given by $[3,8]$

$$
\begin{aligned}
\left|f_{n l}^{\text {ion }}\left(p_{e}, q_{e}\right)\right|^{2}= & \frac{4 p_{e}}{2 \pi} \sum_{L=1}^{\infty} \sum_{l^{\prime}=0}^{\infty} \frac{m_{l}}{2}\left(2 l^{\prime}+1\right)(2 L+1) \\
& \times\left(\begin{array}{ccc}
l & l^{\prime} & L \\
0 & 0 & 0
\end{array}\right)^{2}\left|\int d r r^{2} R_{p_{e} l^{\prime}} R_{n l} j_{L}\left(q_{e} r\right)\right|^{2},
\end{aligned}
$$

where $R_{n l}$ is the radial wave function of the $n, l$ bound state orbital with shell occupancy $m_{l} \leq 2(2 l+1), R_{p_{e} l^{\prime}}$ is the continuum wave function of the electron with momentum $p_{e}$, and $j_{L}$ is the spherical Bessel function of the first kind. [The normalization is $\int d r r^{2} R_{n^{\prime} l^{\prime}} R_{n l}=\delta_{n n^{\prime}} \delta_{l l^{\prime}}$ for bound states and $\int d r r^{2} R_{k l} R_{k^{\prime} l^{\prime}}=(2 \pi) \delta\left(k-k^{\prime}\right) \delta_{l l^{\prime}}$ for states in the continuum.] The $L=0$ term does not induce a transition and is omitted in sum for clarity.

A detailed calculation of $d p_{n, l \rightarrow E_{e}} / d E_{e}$ was presented in Ref. [2] using a fully relativistic formulation. Numerical results were calculated in the dipole approximation, which in the above formulation would amount to expanding $j_{1}\left(q_{e} r\right) \simeq q_{e} r / 3$ and taking the $L=1$ term. In Ref. [2], bound and continuum state wave functions of angular momentum $j=l \pm s$ were generated with the FAC atomic code [50]. Following this approach, we find agreement with their results. Since electron energies remain in the nonrelativistic domain (with small corrections for $n=1$ states in elements with large atomic number), a formulation where differences from spin are unresolved will capture the process to sufficient accuracy. Such approximation has been chosen in Eq. (5) where $l$ labels two spin-degenerate states.

(When the momentum transfer exceeds several $\mathrm{MeV}$, as would be the case for DM with mass above the GeV scale, the nonrelativistic approximation underestimates the form factor [51] and a full treatment becomes necessary.)

The dipole approximation in Eq. (5) results in the following scaling relation in the form factor

$\left|f_{n l}^{\text {ion }}\left(p_{e}, q\right)\right|^{2}=\frac{q^{2}}{q_{0}^{2}} \times\left|f_{n l}^{\text {ion }}\left(p_{e}, q_{0}\right)\right|^{2} \quad$ (dipole approx).

Here, $q_{0}$ is a reference momentum at which the scaling relation holds, i.e., $q_{0} r_{e} \ll 1$, where $r_{e}$ is the electron radius; for xenon atoms, $q_{0} \lesssim 1 \mathrm{keV}$. The scaling-relation breaks down once $q r_{e} \gtrsim 1$ as higher multipole $(L>1)$ contributions in Eq. (5) become important and must be included; in our numerical results we include terms up to $L=15$ using FAC-generated continuum and bound-state wave functions. The general behavior of $\left|f_{n l}^{\text {ion }}\left(p_{e}, q\right)\right|^{2}$ is that it peaks at $q \sim$ $\mathrm{keV}$ before becoming strongly suppressed in the high- $q$ region of tens of $\mathrm{keV}$ and above. This behavior is illustrated in Fig. 1 where the area of validity of Eq. (6) is indicated.

Cross sections.-The prompt ionization resulting from DM-nuclear scattering with nuclear recoil energy $E_{R}=$ $q^{2} / 2 m_{N}$ leads to a double-differential cross section in $E_{R}$ and $E_{e}$. Away from kinematic end points, the cross section can be factorized as the elastic cross section times the electron ionization probability [2], 


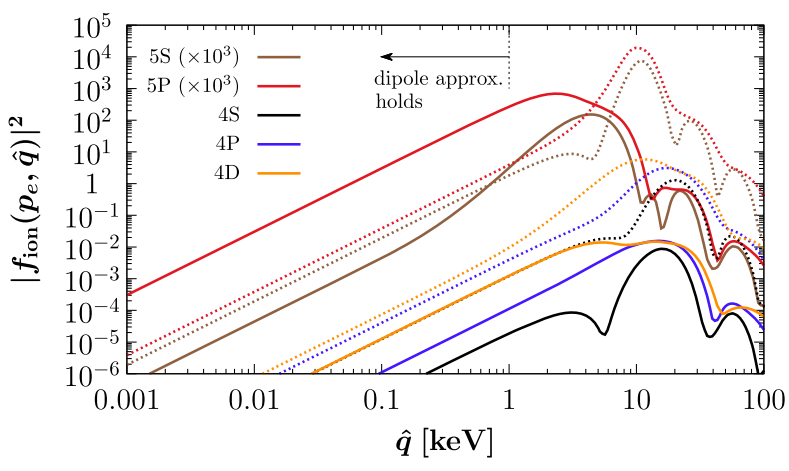

FIG. 1. Ionization form factors for the $n=4$ and $n=5$ shells in xenon as a function of $q$ for $E_{e}=1 \mathrm{eV}(100 \mathrm{eV})$ shown by the solid (dotted) lines. Below $q=1 \mathrm{keV}$, the form factor scales as $q^{2}$ and the dipole approximation in Eq. (6) is valid. At higher momentum transfers, $\left|f_{n l}^{\text {ion }}\left(p_{e}, q\right)\right|^{2}$ tends to peak before becoming strongly suppressed; $\hat{q}=q_{e}$ for Migdal whereas $\hat{q}=q$ for DM-electron scattering.

$$
\frac{d \sigma_{n, l}}{d E_{R} d E_{e}} \simeq \frac{d \sigma}{d E_{R}} \times \frac{1}{2 \pi} \frac{d p_{n, l \rightarrow E_{e}}}{d E_{e}} .
$$

The elastic DM-nucleus recoil cross section is given by

$\frac{d \sigma}{d E_{R}}=\frac{\bar{\sigma}_{n} m_{N}}{2 \mu_{n}^{2} v^{2}}\left[f_{p} Z+f_{n}(A-Z)\right]^{2}\left|F_{\mathrm{DM}}(q)\right|^{2}\left|F_{N}(q)\right|^{2}$,

where $Z$ is the nuclear charge, $f_{p, n}$ are dimensionless DM couplings to the proton (neutron), and $\mu_{n}$ is the DM-nucleon reduced mass. The DM-nucleon cross section is defined as $\bar{\sigma}_{n} \equiv \mu_{n}^{2} \overline{\left|\mathcal{M}_{n}\left(q=q_{0}\right)\right|^{2}} /\left(16 \pi m_{\chi}^{2} m_{n}^{2}\right)$, where $q_{0}=\alpha m_{e}$ is a reference momentum scale. The model dependence in the DM interaction with the nucleus is absorbed into the definition of a form factor,

$\left|F_{\mathrm{DM}}(q)\right|^{2} \equiv \frac{\overline{\left|\mathcal{M}_{n}(q)\right|^{2}}}{\overline{\left|\mathcal{M}_{n}\left(q=q_{0}\right)\right|^{2}}}=\left\{\begin{array}{ll}1 & \text { contact } \\ \frac{m_{V}^{2}+q_{0}^{2}}{m_{V}^{2}+q^{2}} & \text { light med }\end{array}\right.$,

where $\overline{\left|\mathcal{M}_{n}(q)\right|^{2}}$ is the matrix element for scattering on a free nucleon. In the second equality, the two given examples correspond to contact interactions and to the exchange of a vector mediator with mass $m_{V}$ and a reference momentum $q_{0}$. For sub-GeV DM, the momentum transfer does not resolve the nucleus, $q R_{N} \ll 1$, and the nuclear form factor $\left|F_{N}(q)\right|^{2}=1$ to good approximation.

Integrating Eq. (7) over $E_{R}$ then yields the Migdal electron spectrum, where the kinematic limits of $E_{R}$ are fixed by energy conservation. The cross section is then averaged over the galactic velocity distribution of DM, boosted into the laboratory frame of the detector, $g_{\operatorname{det}}(\boldsymbol{v})$ [52], $d\left\langle\sigma_{n, l} v\right\rangle / d E_{e}=\int_{|v|>v_{\min }} d^{3} \boldsymbol{v} g_{\mathrm{det}}(\boldsymbol{v}) d \sigma_{n, l} v / d E_{e}$. Here, $v_{\text {min }}$ is the minimum velocity such that the DM energy is larger than the total energy received by the electron, $v_{\text {min }}=\sqrt{2 \Delta E_{n, l} / \mu_{N}} ;$ and $\Delta E_{n, l}=E_{e}+\left|E_{n, l}\right|$, where $E_{n, l}$ is the binding energy, which we take from Ref. [9].
Finally, the event rate is given by $d R_{n, l} / d E_{e}=$ $N_{T}\left(\rho_{\mathrm{dm}} / m_{\chi}\right) d\left\langle\sigma_{n, l} v\right\rangle / d E_{e}$, where $N_{T}$ is the number of targets per $\mathrm{kg}$ of detector material and $\rho_{\mathrm{dm}} \simeq$ $0.3 \mathrm{GeV} / \mathrm{cm}^{3}$ is the local DM density. The resulting rate as a function of $\Delta E_{n, l}$, summed over all $l$ for the various $n$ shells of xenon, is shown in Fig. 2 (left) for contact interactions (top panel) and light mediator exchange (bottom panel).

The velocity-averaged ionization cross section can be written in a form analogous to the one used in DM-electron scattering [3] by exchanging the integration order between $d^{3} \boldsymbol{v}$ and $d E_{R}\left(=q d q / m_{N}\right)$,

$$
\begin{aligned}
\frac{d\left\langle\sigma_{n, l} v\right\rangle}{d \ln E_{e}}= & \frac{\bar{\sigma}_{n}}{8 \mu_{n}^{2}}\left[f_{p} Z+f_{n}(A-Z)\right]^{2} \int d q\left\{q\left|F_{N}(q)\right|^{2}\right. \\
& \left.\times\left|F_{\mathrm{DM}}(q)\right|^{2}\left|f_{n l}^{\mathrm{ion}}\left(p_{e}, q_{e}\right)\right|^{2} \eta\left[v_{\min }\left(q, \Delta E_{n, l}\right)\right]\right\},
\end{aligned}
$$

where $\eta\left(v_{\min }\right)$ is the usual velocity average of the inverse speed, $\eta\left(v_{\text {min }}\right)=\left\langle(1 / v) \Theta\left(v-v_{\text {min }}\right)\right\rangle_{g_{\text {det }}}$, but now the minimum velocity becomes a function of both $q$ and $\Delta E_{n, l}$, $v_{\min }\left(q, \Delta E_{n, l}\right) \simeq q /\left(2 m_{\chi}\right)+\Delta E_{n, l} / q$, where we have made the approximation $\mu_{N} \simeq m_{\chi}$ in the expression for $v_{\min }$ as is applicable for sub-GeV DM. Comparing Eq. (10) to the cross section for DM-electron scattering [3],

$$
\begin{aligned}
\frac{d\left\langle\sigma_{n, l}^{\mathrm{DM}-\mathrm{e}} v\right\rangle}{d \ln E_{e}}= & \frac{\bar{\sigma}_{e}}{8 \mu_{e}^{2}} \int d q\left\{q\left|F_{\mathrm{DM}}(q)\right|^{2}\left|f_{n l}^{\mathrm{ion}}\left(p_{e}, q\right)\right|^{2}\right. \\
& \left.\times \eta\left[v_{\min }\left(q, \Delta E_{n, l}\right)\right]\right\},
\end{aligned}
$$

exposes the striking similarity of both processes, with a key difference. The DM form factor is now evaluated at the scale $q$ not $q_{e}$ as the electron directly picks up the entire momentum transfer.

Any direct comparison between the two processes is model dependent as it requires specifying both $\bar{\sigma}_{e}$ and $\bar{\sigma}_{n}$. Consider for concreteness the scattering mediated by a dark photon, coupling to electric charge $e$ with a strength $\varepsilon e$. Then,

$$
\bar{\sigma}_{e}=\frac{16 \pi \varepsilon^{2} \alpha \alpha_{D} \mu_{e}^{2}}{\left(q_{0}^{2}+m_{V}^{2}\right)^{2}}, \quad \bar{\sigma}_{p}=\frac{\mu_{p}^{2}}{\mu_{e}^{2}} \bar{\sigma}_{e},
$$

where $\bar{\sigma}_{p}$ is the DM-proton scattering cross section $\left(f_{p}=1, f_{n}=0\right), \alpha_{D}=g_{V}^{2} / 4 \pi$, where $g_{V}$ is the DM-dark photon coupling, and for consistency we define $\bar{\sigma}_{n}=$ $\left(Z^{2} / A^{2}\right) \bar{\sigma}_{p}$. In the expression for the Migdal effect, $\left|F_{N}(q)\right|^{2}$ needs to account for the screening of the nuclear electric potential; see, e.g., Ref. [46]. It is, however, of little relevance for the typically considered momentum transfers, and we will neglect it to exhibit better the parametric relations.

For a dark photon mediator, the ratio of differential cross sections in Eqs. (10) and (11) becomes 

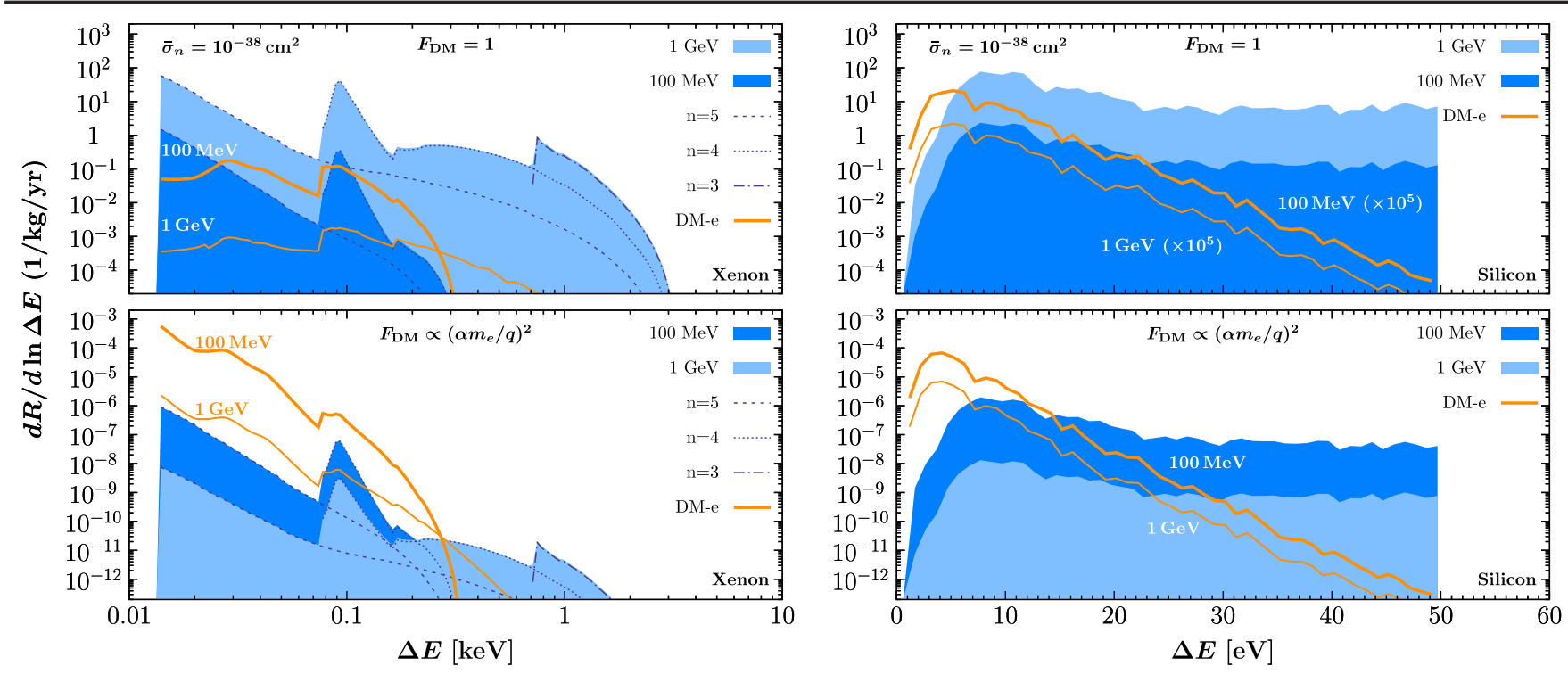

FIG. 2. Left: Light (dark) shaded regions show the cumulative Migdal event rates in xenon for $m_{\chi}=1 \mathrm{GeV}(100 \mathrm{MeV})$ as a function of deposited electronic energy $\Delta E$ for contact interactions (top panel) and light mediator scaling (bottom panel). Individual contributions from the various shells are indicated by the various dashed lines. For the light mediator a reference momentum of $q_{0}=\alpha m_{e}$ and $m_{V}=0$ is chosen. The rates for DM-electron scattering for $m_{\chi}=1 \mathrm{GeV}(100 \mathrm{MeV})$ are shown by the thick (thin) orange solid lines. Right: Same as the left panel, but for silicon and evaluated using our formulation of the Migdal effect in semiconductors. The rates are averaged over a $1 \mathrm{eVenergy} \mathrm{bin.}$

$$
\frac{d\left\langle\sigma_{n, l}^{\text {Migdal }} v\right\rangle /\left(d \ln E_{e} d q\right)}{d\left\langle\sigma_{n, l}^{\mathrm{DM}-e} v\right\rangle /\left(d \ln E_{e} d q\right)}=Z^{2} \times \frac{\left|f_{n l}^{\mathrm{ion}}\left(p_{e}, q_{e}\right)\right|^{2}}{\left|f_{n l}^{\mathrm{ion}}\left(p_{e}, q\right)\right|^{2}} .
$$

At this point, one may be tempted to exploit the dipole scaling relationship in Eq. (6) to conclude that the right-hand side scales as $Z^{2} m_{e}^{2} / m_{N}^{2} \sim 10^{-6}(Z / A)^{2}$, implying that the rate for Migdal ionization is always much smaller than for DMelectron scattering. However, whereas the dipole approximation is always valid for the Migdal effect, since $q_{e} r_{e} \ll 1$, kinematic arguments imply that for DM-electron scattering $\Delta E_{n, l} / v_{\max } \lesssim q \lesssim 2 \mu_{N} v_{\max }$. In this region, $q \gtrsim 1 / r_{e}$ and the dipole scaling breaks down. Inspection of Fig. 1 shows that at large enough momentum transfer the form factors become strongly suppressed, so that at some critical value $q^{\text {crit }}>1 / r_{e},\left|f_{n l}^{\text {ion }}\left(p_{e}, q^{\text {crit }}\right)\right|^{2}=\left|f_{n l}^{\text {ion }}\left(p_{e}, q_{e}^{\text {crit }}\right)\right|^{2} \times Z^{2}($ with $\left.q_{e}^{\text {crit }}=\left(m_{e} / m_{N}\right) q^{\text {crit }}\right)$ is certainly met. Hence, for the differential rates in Eq. (13), DM-electron scattering only dominates over Migdal scattering for $q<q^{\text {crit }}$, while the Migdal effect dominates over DM-electron scattering for higher mass DM. Clearly, both effects need to be taken into account to derive accurate DM constraints. For contact interactions, the Migdal effect dominates for $m_{\chi} \gtrsim\left(q^{\text {crit }}\right)^{2} /\left(2 \Delta E_{n, l}\right)$. For long-range interactions, DM-electron interactions dominate essentially for all masses as there is a bias towards lower momenta introduced by $\left|F_{\mathrm{DM}}(q)\right|^{2}$.

Application to semiconductors. - Until now, all studies of the Migdal effect in semiconductor targets considered isolated atoms. Using our above results and working along the lines of Ref. [22] that previously exposed the connection for DM-electron scattering between isolated atoms and semiconductors, we are in a position to arrive at a rate

equation for Migdal scattering in semiconductors. The replacement to be made in Eq. (10) to obtain the analogous cross section $d\left\langle\sigma_{\text {crystal }} v\right\rangle / d \ln E_{e}$ is

$$
\left|f_{n, l}^{\text {ion }}\left(q_{e}, E_{e}\right)\right|^{2} \rightarrow \frac{8 \alpha m_{e}^{2} E_{e}}{q_{e}^{3}} \times\left|f_{\text {crystal }}\left(q_{e}, E_{e}\right)\right|^{2},
$$

where $\left|f_{\text {crystal }}\left(q_{e}, E_{e}\right)\right|^{2}$ is a dimensionless crystal form factor obtained in Refs. [22,53]. The ionization rate is then given by $d R_{\text {crystal }} / d \ln E_{e}=N_{\text {cell }}\left(\rho_{\chi} / m_{\chi}\right) d\left\langle\sigma_{\text {crystal }} v\right\rangle / d \ln E_{e}$, where $N_{\text {cell }} \equiv M_{\text {target }} / M_{\text {cell }}=N_{T} / 2$ is the number of unit cells in the crystal.

In deriving Eq. (14) we made use of the dipole scaling relation (6). This scaling is only approximate: due to the crystal's electronic structure, there are only discrete values of $q_{e}$ available for a given $E_{e}$. Moreover, because of small $q_{e}$, the Migdal effect favors direct-gap transitions $(\gtrsim 3 \mathrm{eV})$ over indirect-gap transition ( $\gtrsim 1.2 \mathrm{eV})$, affecting the threshold behavior with respect to DM-e scattering. A more refined computation of the crystal form factor at low momentum is warranted and left to Ref. [49]. Here we show a proof of concept of the mapping between the ionization and crystal form factors, and calculate the first projections on $\bar{\sigma}_{n}$ from the Migdal effect in silicon. Since the dipole approximation is valid when $q r_{e} \ll 1$, where $r_{e} \sim 1 /\left(\alpha m_{e}\right)$ in silicon, we anchor our form factors at $q_{0}=0.5 \alpha m_{e}$ and take the average value of $f_{\text {ion }}$ in a neighborhood around $q_{0}$. The QEdark [53] form factors are calculated up to $E_{e}=50 \mathrm{eV}$, which is sufficient for DM-electron scattering. However, from Fig. 2 (right), we see that Migdal scattering extends out to higher values of $E_{e}$ and the truncation at $E_{e}=50 \mathrm{eV}$ underestimates the 


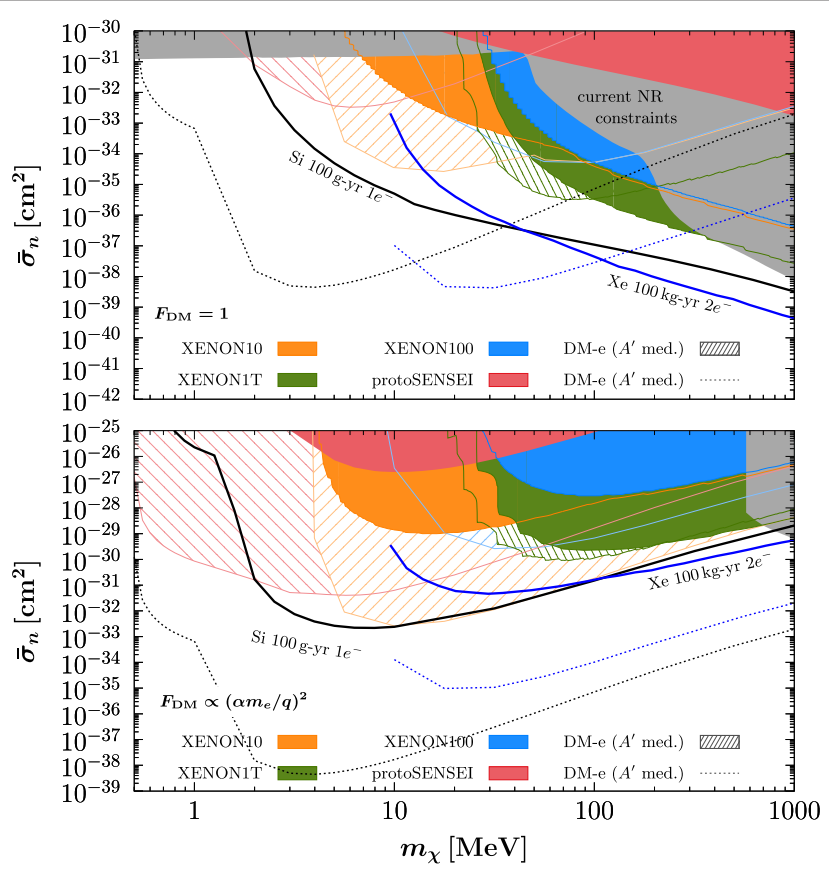

FIG. 3. The $90 \%$ C.L. with $f_{n}=f_{p}=1$ for a heavy (top panel) and light (bottom panel) mediator from XENON10, XENON100, and XENON1T as well as projections for a $100 \mathrm{~g}$ yr silicon detector and LBECA, a $100 \mathrm{~kg}$ yr xenon detector, with 2-electron thresholds. The solid regions correspond to Migdal scattering, while the hatched regions are the translation of DM-electron scattering constraints in a dark photon model. In the top panel, the gray-shaded region corresponds to current constraints on $\bar{\sigma}_{n}$ from CRESST III [63], organic liquid scintillator [64], DarkSide-50 [65], CDEX [6], XENON1T $[60,66]$, and a recast of XENON1T data for cosmicray up scatter [44]. In the bottom panel, the gray-shaded region corresponds to constraints from LUX [4] and Panda-X [67].

rate; in principle, one can combine the form factors calculated with relativistic FAC wave functions, which are sufficiently accurate for $E_{e} \gtrsim 50 \mathrm{eV}$ to obtain a more complete spectrum [49]. Here, we show the constraints using the QEdark form factors only.

Constraints and projections.-We now apply the above results to derive new constraints and projections from direct detection experiments. For xenon detectors, the ionization (S2) signal is obtained by folding $d R / d E_{e}$ with the probability to produce $S 2$ photoelectrons (PE) given a deposited energy $\Delta E_{n l}$ and a detection efficiency $\epsilon(S 2)$, $d R_{n l} / d S 2=\epsilon(S 2) \int d E_{e} P\left(S 2 \mid \Delta E_{n l}\right) d R_{n l} / d E_{e}$. Here, $P\left(S 2 \mid \Delta E_{n l}\right)=\sum_{n_{e}^{\text {surv }}, n_{e}} P\left(S 2 \mid n_{e}^{\text {surv }}\right) P\left(n_{e}^{\text {surv }} \mid n_{e}\right) P\left(n_{e} \mid\left\langle n_{e}\right\rangle\right)$. The number of electrons escaping the interaction point $n_{e}$ are assumed to follow a binomial distribution, $P\left(n_{e} \mid\left\langle n_{e}\right\rangle\right)=$ $\operatorname{binom}\left(n_{e} \mid N_{Q}, f_{e}\right)$ with $N_{Q}=\Delta E_{n l} /(13.8 \mathrm{eV})[54,55]$ trials and a single event probability $f_{e}=\left\langle n_{e}\right\rangle / N_{Q}$; the mean number of electrons $\left\langle n_{e}\right\rangle$ is either modeled following Ref. [8] or, for $\Delta E_{n l}>0.19 \mathrm{keV}$, obtained from the measured charge yield in Ref. [56]. For $P\left(n_{e}^{\text {surv }} \mid n_{e}\right)$, we assume that $80 \%$ $(100 \%)$ of electrons survive the drift in XENON1T/ XENON100 (XENON10). Those electrons induce scintillation at the liquid-gas interface, described by a Gaussian, $P\left(S 2 \mid n_{e}^{\text {surv }}\right)=\operatorname{gauss}\left(S 2 \mid g_{2} n_{e^{\text {surv }}}, \sigma_{S 2}\right)$, with a representative width $\sigma_{S 2}=7 \sqrt{n_{e}^{\text {surv }}}$ [57] and a gain factor $g_{2}=33,20$, and $27 \mathrm{PE} / e^{-}$for XENON1T, XENON100, and XENON10, respectively. For silicon, we use $d R / d n_{e}=\int d E_{e} P\left(E_{e} \mid n_{e}\right) d R / d E_{e}$, where $P\left(E_{e} \mid n_{e}\right)=\delta(1+$ Floor $\left.\left[E_{e} / 3.8 \mathrm{eV}\right]-n_{e}\right)$. We reinterpret the data and efficiencies for XENON10 [58], XENON100 [59], XENON1T [60], and SENSEI [15] to obtain the exclusion boundaries in Fig. 3. We leave to future work calculating the limits from DarkSide-50 [14], CDMS-HVeV [13], and DAMIC at SNOLAB [16]. We also show the $90 \%$ C.L. projections for a future $100 \mathrm{~g}$ yr silicon detector (like SENSEI) with a single-electron threshold, a dark count of $10^{6}$ events for $n_{e}=1$ and no background events for $n_{e} \geq 2$; and a $100 \mathrm{~kg} \mathrm{yr}$ xenon detector (like LBECA) with a two-electron threshold and assuming only neutrino induced backgrounds $[10,61,62]$. The new constraint from XENON10 extends sensitivity to DM-nuclear scattering down to $m_{\chi} \sim 5 \mathrm{MeV}$, and future experiments will reach $\sim 500 \mathrm{keV}$. We also compare the DM-electron scattering limits with the Migdal effect, assuming a dark photon mediator, and converting the limits and projections from $\bar{\sigma}_{e}$ to $\bar{\sigma}_{n}$ using Eq. (12). For contact interactions, the DM-electron scattering limits are stronger for smaller DM masses, but weaker for heavier DM masses, than the Migdal limits. For light mediators, the DMelectron scattering dominates for all masses.

Conclusions.-The prompt electron ionization signal that may accompany DM-nuclear scattering, the "Migdal effect," allows us to extend the sensitivity of noble liquid and semiconductor $\mathrm{DM}$ detectors into the $\mathrm{MeV}$ mass region. In this work, we show that the theoretical description of the process is closely related to DM-electron scattering, and set the lowest DM-mass constraints on DM-nuclear scattering using XENON10 and XENON100 data. Finally, we take the first step towards a concise formulation of the Migdal effect in semiconductors and demonstrate the future potential for both upcoming noble liquid and semiconductor experiments.

We thank R. Budnik and R. Lang for correspondence on the XENON1T results. The work of R. E. and M. S. in this paper is supported by DOE Grant No. DE-SC0017938. R. E. also acknowledges support from the US-Israel Binational Science Foundation under Grant No. 2016153, from the Heising-Simons Foundation under Grant No. 79921, from a subaward for the DOE Grant No. DE-SC0018952, and from Simons Investigator Award No. 623940. J. P. is supported by the "New Frontiers" program of the Austrian Academy of Sciences. We thank the Erwin Schrödinger International Institute for hospitality while this work was completed. T.-T. Y. also thanks the hospitality of the CERN theory department where part of this work was completed.

Note added.—Recently, Ref. [68] appeared which investigates similar topics for the isolated atom case. 
*rouven.essig@stonybrook.edu

†josef.pradler@oeaw.ac.at

${ }^{\ddagger}$ mukul.sholapurkar@stonybrook.edu

§tientien@uoregon.edu

[1] C. Kouvaris and J. Pradler, Phys. Rev. Lett. 118, 031803 (2017).

[2] M. Ibe, W. Nakano, Y. Shoji, and K. Suzuki, J. High Energy Phys. 03 (2018) 194.

[3] R. Essig, J. Mardon, and T. Volansky, Phys. Rev. D 85, 076007 (2012).

[4] D. S. Akerib et al. (LUX Collaboration), Phys. Rev. Lett. 122, 131301 (2019).

[5] E. Armengaud et al. (EDELWEISS Collaboration), Phys. Rev. D 99, 082003 (2019).

[6] Z. Z. Liu et al. (CDEX Collaboration), Phys. Rev. Lett. 123, 161301 (2019).

[7] E. Aprile et al. (XENON Collaboration), Phys. Rev. Lett. 123, 241803 (2019).

[8] R. Essig, A. Manalaysay, J. Mardon, P. Sorensen, and T. Volansky, Phys. Rev. Lett. 109, 021301 (2012).

[9] R. Essig, T. Volansky, and T.-T. Yu, Phys. Rev. D 96, 043017 (2017).

[10] J. Tiffenberg, M. Sofo-Haro, A. Drlica-Wagner, R. Essig, Y. Guardincerri, S. Holland, T. Volansky, and T.-T. Yu (SENSEI Collaborations), Phys. Rev. Lett. 119, 131802 (2017).

[11] R. K. Romani et al., Appl. Phys. Lett. 112, 043501 (2018).

[12] M. Crisler, R. Essig, J. Estrada, G. Fernandez, J. Tiffenberg, M. Sofo haro, T. Volansky, and T.-T. Yu (SENSEI Collaboration), Phys. Rev. Lett. 121, 061803 (2018).

[13] R. Agnese et al. (SuperCDMS Collaboration), Phys. Rev. Lett. 121, 051301 (2018); 122, 069901(E) (2019).

[14] P. Agnes et al. (DarkSide Collaboration), Phys. Rev. Lett. 121, 111303 (2018).

[15] O. Abramoff et al. (SENSEI Collaboration), Phys. Rev. Lett. 122, 161801 (2019).

[16] A. Aguilar-Arevalo et al. (DAMIC Collaboration), Phys. Rev. Lett. 123, 181802 (2019).

[17] M. Settimo (DAMIC Collaboration), in Proceedings of the 53rd Rencontres de Moriond on QCD and High Energy Interactions (Moriond QCD 2018) La Thuile, Italy, 2018 (2018).

[18] J. D. Vergados and H. Ejiri, Phys. Lett. B 606, 313 (2005).

[19] C. C. Moustakidis, J. D. Vergados, and H. Ejiri, Nucl. Phys. B727, 406 (2005).

[20] H. Ejiri, C. C. Moustakidis, and J. D. Vergados, Phys. Lett. B 639, 218 (2006).

[21] R. Bernabei et al., Int. J. Mod. Phys. A 22, 3155 (2007).

[22] R. Essig, M. Fernandez-Serra, J. Mardon, A. Soto, T. Volansky, and T.-T. Yu, J. High Energy Phys. 05 (2016) 046.

[23] P. W. Graham, D. E. Kaplan, S. Rajendran, and M. T. Walters, Phys. Dark Universe 1, 32 (2012).

[24] H. An, M. Pospelov, J. Pradler, and A. Ritz, Phys. Lett. B 747, 331 (2015).

[25] E. Aprile et al. (XENON100 Collaboration), Phys. Rev. D 90, 062009 (2014); 95, 029904(E) (2017).

[26] S. K. Lee, M. Lisanti, S. Mishra-Sharma, and B. R. Safdi, Phys. Rev. D 92, 083517 (2015).

[27] Y. Hochberg, Y. Zhao, and K. M. Zurek, Phys. Rev. Lett. 116, 011301 (2016).
[28] Y. Hochberg, M. Pyle, Y. Zhao, and K. M. Zurek, J. High Energy Phys. 08 (2016) 057.

[29] A. Aguilar-Arevalo et al. (DAMIC Collaboration), Phys. Rev. Lett. 118, 141803 (2017).

[30] I. M. Bloch, R. Essig, K. Tobioka, T. Volansky, and T.-T. Yu, J. High Energy Phys. 06 (2017) 087.

[31] G. Cavoto, E. N. M. Cirillo, F. Cocina, J. Ferretti, and A. D. Polosa, Eur. Phys. J. C 76, 349 (2016).

[32] S. Derenzo, R. Essig, A. Massari, A. Soto, and T.-T. Yu, Phys. Rev. D 96, 016026 (2017).

[33] R. Essig, J. Mardon, O. Slone, and T. Volansky, Phys. Rev. D 95, 056011 (2017).

[34] Y. Hochberg, Y. Kahn, M. Lisanti, C. G. Tully, and K. M. Zurek, Phys. Lett. B 772, 239 (2017).

[35] Y. Hochberg, T. Lin, and K. M. Zurek, Phys. Rev. D 94, 015019 (2016).

[36] Y. Hochberg, T. Lin, and K. M. Zurek, Phys. Rev. D 95, 023013 (2017).

[37] R. Budnik, O. Chesnovsky, O. Slone, and T. Volansky, Phys. Lett. B 782, 242 (2018).

[38] P. C. Bunting, G. Gratta, T. Melia, and S. Rajendran, Phys. Rev. D 95, 095001 (2017).

[39] G. Cavoto, F. Luchetta, and A. D. Polosa, Phys. Lett. B 776, 338 (2018).

[40] S. Fichet, Phys. Rev. Lett. 120, 131801 (2018).

[41] S. Knapen, T. Lin, M. Pyle, and K. M. Zurek, Phys. Lett. B 785, 386 (2018).

[42] Y. Hochberg, Y. Kahn, M. Lisanti, K. M. Zurek, A. G. Grushin, R. Ilan, S. M. Griffin, Z.-F. Liu, S. F. Weber, and J. B. Neaton, Phys. Rev. D 97, 015004 (2018).

[43] M. J. Dolan, F. Kahlhoefer, and C. McCabe, Phys. Rev. Lett. 121, 101801 (2018).

[44] T. Bringmann and M. Pospelov, Phys. Rev. Lett. 122, 171801 (2019).

[45] Y. Ema, F. Sala, and R. Sato, Phys. Rev. Lett. 122, 181802 (2019).

[46] T. Emken, R. Essig, C. Kouvaris, and M. Sholapurkar, J. Cosmol. Astropart. Phys. 09 (2019) 070.

[47] N. F. Bell, J. B. Dent, J. L. Newstead, S. Sabharwale, and T. J. Weiler, arXiv:1905.00046.

[48] C. Cappiello and J. F. Beacom, Phys. Rev. D 100, 103011 (2019).

[49] R. Essig, L. Matzi, J. Pradler, M. Sholapurkar, and T.-T. Yu (to be published).

[50] M. F. Gu, Can. J. Phys. 86, 675 (2008).

[51] B. M. Roberts, V. A. Dzuba, V. V. Flambaum, M. Pospelov, and Y. V. Stadnik, Phys. Rev. D 93, 115037 (2016).

[52] J. D. Lewin and P.F. Smith, Astropart. Phys. 6, 87 (1996).

[53] “QEdark," http://ddldm.physics.sunysb.edu/.

[54] C. E. Dahl, The physics of background discrimination in liquid xenon, and first results from Xenon10 in the hunt for WIMP dark matter, Ph.D. thesis, Princeton University, 2009.

[55] D. S. Akerib et al. (LUX Collaboration), Phys. Rev. D 95, 012008 (2017).

[56] D. S. Akerib et al. (LUX Collaboration), Phys. Rev. D 96, 112011 (2017).

[57] E. Aprile et al. (XENON100 Collaboration), J. Phys. G 41, 035201 (2014). 
[58] J. Angle et al. (XENON10 Collaboration), Phys. Rev. Lett. 107, 051301 (2011); 110, 249901(E) (2013).

[59] E. Aprile et al. (XENON Collaboration), Phys. Rev. D 94, 092001 (2016); 95, 059901(E) (2017).

[60] E. Aprile et al., Phys. Rev. Lett. 123, 251801 (2019).

[61] R. Essig, M. Sholapurkar, and T.-T. Yu, Phys. Rev. D 97, 095029 (2018).

[62] M. Battaglieri et al., in U.S. Cosmic Visions: New Ideas in Dark Matter College Park, MD, USA, 2017 [arXiv: 1707.04591].
[63] A. H. Abdelhameed et al. (CRESST Collaboration), Phys. Rev. D 100, 102002 (2019).

[64] J. I. Collar, Phys. Rev. D 98, 023005 (2018).

[65] P. Agnes et al. (DarkSide Collaboration), Phys. Rev. Lett. 121, 081307 (2018).

[66] E. Aprile et al. (XENON Collaboration), Phys. Rev. Lett. 121, 111302 (2018).

[67] X. Ren et al. (PandaX-II Collaboration), Phys. Rev. Lett. 121, 021304 (2018).

[68] D. Baxter, Y. Kahn, and G. Krnjaic, arXiv:1908.00012. 